\title{
Prioridades Axiológicas e Uso de Preservativo
}

\author{
Alvaro Tamayo $^{1}$ \\ Adilce Lima \\ Juliana Marques \\ Larissa Martins \\ Universidade de Brasilia
}

\begin{abstract}
Resumo
O Inventário de Valores de Schwartz foi administrado a 300 estudantes universitários com o objetivo de estudar a relação entre as prioridades axiológicas e a freqüência de uso de preservativo no seu relacionamento sexual. Os dez tipos motivacionais de valores foram correlacionados com a variável dependente. A curva representando a relação entre as prioridades axiológicas e o uso de preservativo foi sinusóide mas não simétrica. Hedonismo, autodeterminação e estimulação correlacionaram-se positivamente com o uso de preservativo enquanto que tradição e conformidade correlacionaram-se negativamente. A correlação com universalismo e benevolência foi próxima de zero. Os resultados da regressão múltipla confirmaram que hedonismo, autodeterminação e estimulação são os preditores axiológicos mais fortes do uso de preservativo pelos estudantes universitários. Palavras-chave: Valores; preservativo; AIDS; Inventário de Valores de Schwartz.
\end{abstract}

\section{Value Priorities and the Use of Condom}

\begin{abstract}
The Schwartz Value Inventory was applied to 300 university students in order to study the relationship between value priorities and the frequency with which they use the condom in their sexual relations. The ten motivational types of values were correlated with the dependent variable. The curve relating value priorities with the use of preservative was sinusoidal, but not symmetrical. Hedonism, self-determination and stimulation correlated positively with condom use while tradition and correlated negatively. The correlation with universalism and benevolence was near zero. The results of multiple regression confirmed that hedonism, self-determination and stimulation are the strongest predictors of the university students' use of condom.

Keywords: Values; condom; AIDS; Schwartz Values Inventory.
\end{abstract}

A preocupação crescente com o avance da AIDS no mundo e, particularmente, nos países em desenvolvimento, tem dado origem a numerosos estudos visando identificar fatores preditivos da adoção de comportamentos preventivos. Segundo Bayés (1992), os comportamentos preventivos são: castidade absoluta, relações sexuais exclusivamente monógamas com parceiro fiel e não contaminado, práticas sexuais sem penetração e relações sexuais com penetração mas sempre com preservativo. Por motivos evidentes, as campanhas de prevenção à AIDS têm focalizado prioritariamente o uso regular e adequado de preservativo no relacionamento sexual. A importância que ocupa este comportamento nos programas preventivos implica na compreensão o mais completa possível do mesmo por parte dos especialistas.

Apesar da insistência dos programas preventivos no uso de preservativo, este comportamento parece não ser muito generalizado. Fisher e Misovich (1990) encontraram,

\footnotetext{
${ }^{1}$ Endereço para correspondência: SHIN, QI 3, conjunto 4, casa 2 - 71.505 240, Brasília - Fone: (61) 368-4019. E-mail: tamayo@unb.br
}

com uma amostra de estudantes universitários dos Estados Unidos, que $64 \%$ dos sujeitos relataram ter praticado sexo vaginal sem preservativo, $70 \%$ praticaram sexo oral sem proteção e $6 \%$ sexo anal sem preservativo. No Brasil, Arruda, Morris e Rutenberg (1987), em pesquisa realizada com amostra nacional, encontraram que o preservativo é muito pouco utilizado. De todas as mulheres em idade fértil e com vida sexual ativa entrevistadas, somente $1,1 \%$ revelaram que o parceiro fazia uso regular do preservativo. Estudos posteriores encontraram dados similares. A pesquisa sobre saúde familiar no Nordeste do Brasil (Ferraz, Ferreira, Rutenberg \& Soares, 1992) mostrou que 39,1\% das mulheres em idade fértil estavam usando algum método moderno de anticoncepção. Destas apenas 1,1\% relatou o uso regular de preservativo pelo parceiro. Quanto à intenção de uso futuro, o preservativo foi apontado como método preferido por $2,2 \%$ de todas as que relataram desejo de utilizar algum método anticonceptivo.

Uma análise avaliativa dos programas preventivos revela que os efeitos dessas campanhas ficam muito aquém dos resultados previstos e esperados. A relação entre 
informação e prática preventiva abrange múltiplas variáveis de natureza variada e em estreita interação (Gimenes e colaboradores, 1996). Vários pesquisadores têm mostrado que o uso de preservativo é um comportamento bastante complexo que implica em variáveis pessoais, interpessoais e situacionais (HelwegLarsen \& Collins, 1994). Como afirmam Bryan, Aiken e West (1999, p.285): "Enquanto cientistas do comportamento, precisamos compreender o que estamos pedindo às pessoas para fazer antes que possamos esperar compreender porque elas seguem e, mais importante ainda, porque não seguem nossas recomendações". É de capital importância, portanto, estudar os múltiplos determinantes do uso de preservativo no relacionamento sexual que, no caso da AIDS, constitui o comportamento preventivo por excelência. Como afirma Shayer (1994, p.14), "ainda se sabe muito pouco acerca dos fatores psicossociais que podem predizer os comportamentos preventivos relacionados à AIDS em geral e ao uso de preservativos, em particular".

Várias pesquisas têm mostrado a existência de crenças e atitudes negativas em relação ao uso de preservativo, no sentido que ele quebra a harmonia e o ritmo do encontro sexual (Wilson, Manual \& Lavelle, 1991) e afeta negativamente a disposição sexual (Campbell, Peplau \& DeBro, 1992). Além disso, não pode ser negligenciada a preocupação da pessoa em relação à reação do parceiro diante da sugestão do uso de camisinha e os possíveis efeitos desta proposta sobre o andamento do encontro sexual. Pesquisas empíricas mostram que estas variáveis têm grande impacto sobre a decisão do uso de preservativo, possivelmente a sua influência é maior do que a de variáveis psicológicas, como as atitudes diante do uso de preservativo e a própria suscetibilidade percebida de contrair a doença (Helwet-Larsen \& Collins, 1994; Walter, Vaughn, Ragin, Cohall \& Kasen, 1994).

Uma barreira para o uso de preservativo surge na própria percepção que as pessoas têm deste comportamento e daqueles que o exibem. Recentemente, Bryan e cols. (1999) encontraram que a proposta de usar preservativo no encontro sexual gera percepções positivas e negativas. O estudante universitário masculino estadounidense que sugere à sua parceira o uso de preservativo é percebido como sendo mais simpático e maduro do que aquele que não faz tal proposta, por outra parte, ele é também percebido como sendo menos romântico e menos excitante. Além disso, os estudantes do sexo masculino acreditam que sugerir o uso de preservativo no encontro sexual reduz as probabilidades de relacionamento sexual, convicção que não é compartilhada pelas mulheres. Hynie e Lydon (1995) observaram que as mulheres que, no contexto da interação sexual, fornecem o preservativo são percebidas como menos simpáticas e atraentes do que aquelas que tinham relacionamento sexual sem proteção ou cujo parceiro tinha preservativo. Correlatos intrapsíquicos do uso de preservativo, tais como atitudes diante do preservativo e crenças sobre a sua eficiência, têm sido observados por vários pesquisadores (Bryan, Aiken \& West, 1996; Wulfert \& Wan, 1993).

Valdisserri e colaboradores (1988), num estudo retrospectivo com uma amostra de sujeitos homossexuais e bissexuais, encontraram como antecedentes do uso de preservativo no sexo anal, o tipo de relacionamento (monogâmico e não anônimo versus múltiplo e/ou anônimo), o grau de aceitabilidade do preservativo, e o uso de álcool e/ou de drogas. Este último comportamento foi também confirmado como antecedente do uso de preservativo pelos resultados obtidos na pesquisa realizada por Kelly, Lawrence e Brasfield (1991). Além disso, os autores identificaram também como preditores do uso de preservativo o número de parceiros sexuais e a prática prévia de se engajar em sexo anal desprotegido.

Emmons, Joseph, Kressler, Montgomery e Ostrow, (1986) estudaram o valor preditivo de variáveis psicológicas, tais como conhecimento sobre a doença, percepção de vulnerabilidade e crenças relacionadas com a eficácia do sistema de saúde. O conhecimento relacionado à AIDS foi a única variável preditiva do uso de preservativo. $\mathrm{O}$ valor preditivo do conhecimento da doença foi confirmado por Thurman e Franklin (1990). Os resultados da pesquisa de Roscoe e Kruger (1990) contestam, porém, a importância do conhecimento sobre a doença como determinante do uso de preservativo. Os resultados revelaram que, apesar de um alto nível de informações sobre a doença, apenas $6,6 \%$ das mulheres e $10 \%$ dos homens usavam preservativo como método de prevenção. Mais uma variável psicológica, o medo personalizado, isto é, a percepção da susceptibilidade pessoal de contaminação, foi identificada como antecedente do uso de preservativo (Thurman \& Franklin, 1990).

No Brasil, Shayer (1994), com amostra de 765 estudantes universitários de primeiro ano, estudou os seguintes fatores preditivos da freqüência de uso de preservativo: disponibilidade do preservativo, grau de informação sobre a saúde e o comportamento sexual do novo parceiro, freqüência de relações sexuais nos seis meses anteriores, número de parceiros sexuais nos seis meses anteriores, freqüência de sexo oral ativo e dois conjuntos de barreiras percebidas ao uso do preservativo. 
Das sete variáveis estudadas, a disponibilidade de preservativo na hora da relação sexual foi o preditor mais importante. Esta variável sozinha explicou mais de $26 \%$ da variância. $O$ valor preditivo das outras seis variáveis foi insignificante. Destas, a mais importante foi o grau de informação sobre a saúde e o comportamento sexual do novo parceiro, que explicou $2 \%$ da variância.

Embora as pesquisas acima discutidas não sejam metodologicamente homogêneas e as amostras tenham sido extraídas de contextos culturais diferentes, os seus resultados apontam para a complexidade do comportamento estudado, influenciado por múltiplas variáveis. Os resultados das pesquisas realizadas na área revelam como principais preditores do uso de preservativo nas relações sexuais a aceitabilidade do mesmo, o medo de contaminação, o uso de álcool e/ou drogas, o número de parceiros, o medo personalizado de contaminação, a informação sobre a saúde e o comportamento sexual de um novo parceiro e, particularmente, a disponibilidade de preservativo no momento do relacionamento sexual. As conclusões das investigações apontam para a necessidade urgente de ampliar a pesquisa nesta área. Shayer (1994) finaliza a sua investigação, afirmando que "é preciso ampliar, não só a natureza das variáveis estudadas, como também, diversificar os tipos de metodologia a serem utilizados" (p. 88).

Nesta pesquisa estudou-se a relação entre as prioridades axiológicas da pessoa e a freqüência de uso de preservativo nas relações sexuais. Os autores não têm informação de nenhuma pesquisa empírica publicada em torno a esta problemática. $\mathrm{O}$ uso de preservativo no relacionamento sexual não é um comportamento que acontece num vácuo social e cultural mas num contexto interpessoal carregado de valores, de crenças, de incertezas e de expectativas. As prioridades axiológicas expressam concepções geralmente compartilhadas de aquilo que é bom para o indivíduo e para a sociedade. Os valores são definidos como princípios que orientam a vida do indivíduo (Tamayo \& Schwartz, 1993). Neste sentido, eles possuem várias funções importantes que são relevantes para as opções do indivíduo e para a compreensão do seu comportamento. Vários pesquisadores têm estudado recentemente a relação entre as prioridades axiológicas das pessoas e os mais variados tipos de comportamento, tais como a prontidão para estabelecer contato social com grupos minoritários (Sagiv \& Schwartz, 1995), o comportamento eleitoral (Schwartz, 1996), as maneiras de abordar e de reagir aos outros em situação de negociação (De Dreu \& Van Lange, 1995), o comportamento religioso (Schwartz \& Huismans, 1995), a atração percebida de alternativas de ação apresentadas ao indivíduo, bem como a própria opção realizada pelo sujeito (Feather, 1995), o comportamento dos consumidores (Kahle, 1996) e as atitudes raciais (Biernat, Theno \& Crandall, 1996). As prioridades axiológicas das pessoas influenciam direta ou indiretamente o uso de preservativo no relacionamento sexual? Este foi o problema estudado na presente pesquisa.

Como afirma Rokeach (1973, p. 122): ... “O conhecimento dos valores de uma pessoa nos deveria permitir predizer como ela se comportará em diversas situações experimentais e da vida real". Determinar o valor preditivo dos valores e, particularmente, estabelecer a sua relação funcional com o comportamento e com as atitudes tem constituído um desafio para os pesquisadores. Os valores podem ser considerados como determinantes do comportamento? Mais especificamente, eles podem ser considerados como preditores de comportamentos concretos, tais como o uso de preservativo? As conseqüências do uso do preservativo são relevantes para a obtenção de objetivos motivacionais inerentes às prioridades axiológicas do indivíduo?

Um sistema de valores, segundo Rokeach (1973, p. 551) é "nada mais do que uma disposição hierárquica de valores, uma classificação ordenada de valores ao longo de um contínuo de importância”. A organização hierárquica de valores pressupõe que o indivíduo não se relaciona com o mundo físico e social como um observador que assiste a um espetáculo, mas como um ator que participa, que toma partido, que se envolve nele. Os valores implicam necessariamente uma preferência, uma distinção entre o que é importante para o indivíduo e o que é secundário, entre o que tem valor e o que não tem. Assim, na essência mesma dos valores parece estar presente a sua relação com o comportamento. Do ponto de vista teórico, não existe dúvida da influência determinante dos valores sobre o comportamento. Segundo Rokeach (1973), os valores são os principais fatores que influenciam e determinam o comportamento social. Segundo ele, isto explica o interesse da psicologia social no estudo dos valores.

Do ponto de vista empírico, a relação entre os valores e o comportamento tem sido objeto de numerosas pesquisas. Rokeach e vários outros pesquisadores estudaram o impacto dos valores sobre os mais variados comportamentos, tais como: afiliação a organizações de defesa dos direitos humanos (Rokeach, 1973), participação em demonstrações a favor dos direitos humanos (Rokeach, 1973), freqüência à igreja (Rokeach, 1969), colagem em sala de aula (Homant \& Rokeach, 1970), devolução de um lápis distribuído para responder 
um questionário (Shotland \& Berger, 1970), escolha de uma determinada área de estudos (Feather, 1970) e identificação com o estilo de vida hippie (Rokeach, 1973). Todas estas pesquisas têm em comum o estudo do impacto de um ou mais valores sobre o comportamento. Desta forma, os valores a serem relacionados com um comportamento determinado eram escolhidos pelo pesquisador através de observações ou de simples intuição, sem uma abordagem global ou de tipo fatorial. Em outras pesquisas a abordagem era ainda mais exploratória. Listas de valores eram relacionadas com outras variáveis e depois eram verificadas e discutidas as correlações significativas que surgiam, por exemplo, com a qualidade de ensino (Greenstein, 1976), com a raça e nacionalidade (Rokeach, 1973). Os paradigmas de pesquisa utilizados eram conseqüência da própria teoria dos valores dominante na época, que não apresentava uma organização estrutural dos valores. Rokeach (1973) dividia os valores em terminais e instrumentais. Os primeiros referiam-se a estados de existência desejáveis e, os segundos a comportamentos desejáveis. Os valores terminais subdividiam-se em pessoais e sociais e os instrumentais em morais e de competência. Esta classificação, porém, era meramente teórica e não oferecia alternativas práticas para o estudo do impacto dos valores sobre o comportamento. Além disso, a própria medida o comportamento parece ser orientado, não pela prioridade dada a um único valor, mas por múltiplos e diversos valores (Rokeach, 1973).

No final da década de 80 Schwartz e Bilsky (1987) lançaram as bases de uma teoria estrutural dos valores que foi posteriormente desenvolvida e verificada através de pesquisa intercultural. Segundo Schwartz (1992), os valores expressam as metas motivacionais do indivíduo. "O conteúdo fundamental que diferencia os valores entre si é o tipo de meta motivacional que expressam" (Schwartz, 1996). Assim, o estudo das prioridades axiológicas refere-se ao estudo de tipos motivacionais de valores (Schwartz, 1992; Tamayo, 1994; Tamayo \& Schwartz, 1993). Dez tipos motivacionais de valores foram deduzidos a partir das exigências básicas do ser humano, a saber, 1) necessidades biológicas do organismo, 2) necessidades sociais relativas à regulação das interações interpessoais e 3) necessidades sócio-institucionais referentes à sobrevivência e bem-estar dos grupos.

Dez tipos motivacionais de valores (TMV) foram deduzidos teoricamente e, posteriormente, verificados empiricamente em diversas culturas. Cada um deles expressa uma tendência motivacional própria e é, em conseqüência, constituído por valores que têm metas e interesses comuns ou altamente similares (Schwartz, 1992). Os TMV são apresentados e definidos na Tabela 1.

Tabela 1. Tipos Motivacionais de Valores

\begin{tabular}{|c|c|c|}
\hline Tipos & Metas & Serve interesses \\
\hline Hedonismo & Prazer e gratificação sensual para si mesmo & Individuais \\
\hline Realização & $\begin{array}{l}\text { O sucesso pessoal obtido através de uma demonstração } \\
\text { de competência }\end{array}$ & Individuais \\
\hline Poder social & Controle sobre pessoas e recursos, prestígio & Individuais \\
\hline Autodeterminação & Independência de pensamento, ação e opção & Individuais \\
\hline Estimulação & Excitação, novidade, mudança, desafio & Individuais \\
\hline Conformidade & $\begin{array}{l}\text { Controle de impulsos e ações que podem violar } \\
\text { normas sociais ou prejudicar os outros }\end{array}$ & Coletivos \\
\hline Tradição & Respeito e aceitação dos ideais e costumes da sociedade & Coletivos \\
\hline Benevolência & Promoção do bem-estar das pessoas íntimas & Coletivos \\
\hline Segurança & $\begin{array}{l}\text { Integridade pessoal, estabilidade da sociedade, do } \\
\text { relacionamento e de si mesmo }\end{array}$ & Mistos \\
\hline Universalismo & $\begin{array}{l}\text { Tolerância, compreensão e promoção do bem-estar de } \\
\text { todos e da natureza }\end{array}$ & Mistos \\
\hline
\end{tabular}

dos valores, que era de tipo ordinal, dificultava uma definição estrutural dos mesmos.

As abordagens mencionadas para o estudo do impacto dos valores sobre o comportamento fornecem resultados muito fragmentários e, além disso, são dificilmente sustentáveis do ponto de vista teórico já que
A relação entre os tipos motivacionais é dinâmica. Ela foi verificada através do método da Análise do Espaço Menor (Smallest Space Analysis) que é uma técnica indicada para a análise estrutural da similaridade de dados. Através dela os valores são representados como pontos no espaço multidimensional, de tal forma que as distâncias entre 
eles expressam as relações empíricas entre os valores, determinadas a partir das correlações entre os seus graus de importância, de acordo com as respostas dos sujeitos. A Figura 1 ilustra a relação dinâmica entre os tipos motivacionais de valores. Os cinco tipos de valores que expressam interesses individuais (autodeterminação, estimulação, hedonismo, realização e poder social) ocupam, no espaço multidimensional, uma área contígua que é oposta àquela reservada aos três conjuntos de valores que expressam primariamente interesses coletivos (benevolência, tradição e conformidade). Os tipos motivacionais segurança e universalismo, constituídos por valores que expressam interesses tanto individuais como coletivos, são opostos e situam-se nas fronteiras destas duas áreas (Tamayo \& Schwartz, 1993). Schwartz e Bilsky $(1987,1990)$ postulam compatibilidade entre os tipos de valores que são adjacentes no espaço multidimensional (por exemplo, estimulação e hedonismo, tradição e conformidade) e conflito entre os tipos de valores situados em direções opostas (exemplo: estimulação e conformidade, hedonismo e tradição). A busca simultânea de valores pertencentes a áreas adjacentes é compatível porque esse tipo de valores está ao serviço de um mesmo interesse. Desta forma, "as ações tomadas no perseguimento de um tipo de valores têm conseqüências psicológicas, práticas e sociais que podem ser conflituosas ou compatíveis com a perseguição de outro tipo de valores" (Sagiv \& Schwartz, 1995, p. 438).

\section{Autotranscendência}

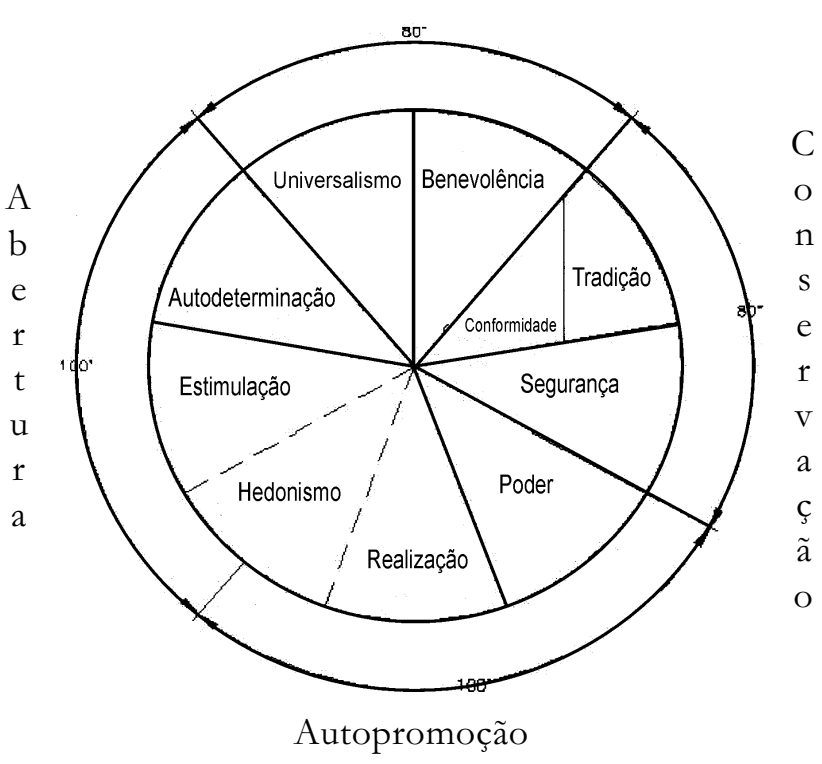

Figura 1. Estrutura bidimensional dos valores
A relação estrutural básica entre os valores e entre os tipos motivacionais por eles constituídos, pode ser sintetizada através de duas dimensões bipolares, já verificadas empiricamente, tanto no Brasil (Tamayo, 1994; Tamayo \& Schwartz, 1993) como no exterior (Schwartz, 1992, 1994). A primeira, "abertura à mudança versus conservação", ordena os valores com base na motivação da pessoa a seguir os seus próprios interesses intelectuais e afetivos através de caminhos incertos e ambíguos, por oposição à tendência a preservar o status quo e a segurança que ele gera no relacionamento com os outros e com as instituições. Esta dimensão expressa o conflito entre as tendências das pessoas a sua independência de pensamento e ação que valoriza a inovação e a mudança em oposição à aceitação e comprometimento com o status quo. Teoricamente, situam-se, num dos pólos deste eixo, os valores relativos aos tipos motivacionais "estimulação" e "autodeterminação" e, no outro, os referentes aos tipos "segurança", "conformidade" e "tradição". A segunda dimensão, "autopromoção versus autotranscendência", apresenta, num dos extremos, os valores relativos aos tipos motivacionais "poder", "realização" e "hedonismo" e, no outro, os valores de universalismo e benevolência. Este eixo ordena os valores com base na motivação da pessoa para promover os seus próprios interesses mesmo às custas dos outros, por oposição a transcender as suas preocupações egoístas e promover o bem-estar dos outros e da natureza (Figura 1).

No contexto desta nova abordagem tem mais sentido estudar o impacto dos valores sobre o comportamento. Uma característica importante do modelo acima apresentado é que ele postula análises de prioridades axiológicas múltiplas e concorrentes, permitindo o estudo da relação dos valores com o comportamento de forma integrada (Schwartz, 1996) A relação entre os valores e qualquer atitude ou comportamento tende a ser associada de forma similar a tipos motivacionais adjacentes na estrutura circular. Além disso, as correlações com as atitudes e com o comportamento diminuem monotonicamente ao longo dos tipos motivacionais nas direções horária ou antihorária da estrutura circular (Figura 1), desde o tipo motivacional mais positivamente correlacionado até o menos positivamente associado. Dessarte, as relações teóricas entre as prioridades axiológicas e o comportamento descrevem uma curva sinusóide. Ao estudar, portanto, o impacto dos valores sobre o uso de preservativo no relacionamento sexual, estar-se-á relacionando todo o conjunto das prioridades axiológicas do indivíduo com o mencionado comportamento. 


\section{Método}

\section{Amostra}

A amostra foi composta por 310 estudantes da Universidade de Brasília, dos dois sexos, com idade média de 23,22 anos $(d p=5,02)$ e sendo a maioria $(88 \%)$ solteiros e de religião católica. Todos os sujeitos investigados tinham vida sexual ativa, mas não necessariamente regular.

\section{Instrumento}

O questionário para a pesquisa compreendia três partes: 1) o Inventário de Valores de Schwartz (Tamayo \& Schwartz, 1993; 2) a avaliação da freqüência pessoal de uso de camisinha no relacionamento sexual, avaliada através de cinco alternativas: nunca, menos da metade das vezes, metade das vezes, mais da metade das vezes e todas as vezes (Shayer, 1994). Além disso, quatro itens, seguidos de uma escala de cinco pontos, relativos à opinião dos sujeitos sobre o uso de preservativo no relacionamento sexual ("o preservativo diminui a sensação sexual", "falarpara o(a) parceiro(a) que você quer usarpreservativo, demostra falta de confiança nele (a)", "as pessoas que prezam a sua saúde devem usar preservativo com regularidade", "os preservativos custam caro"; e, 3) uma série de perguntas relativas a dados pessoais, tais como idade, gênero e religião.

\section{Procedimento}

O questionário para a pesquisa foi aplicado individualmente, na própria universidade, em locais apropriados para esta atividade, tais como sala de aula ou de reuniões, biblioteca, e laboratórios.

\section{Análise dos Dados}

Foram calculadas correlações bivariadas entre cada um dos TMV e a freqüência de uso de preservativo, de acordo com a teoria que postula uma curva sinusóide para a relação entre as prioridades axiológicas e o comportamento (Schwartz, 1996). A análise de regressão múltipla stepwise (entrada $<0,01)$ e $F$ ( saída $<0,05$ ), tendo como variável critério a freqüência de uso de preservativo e como preditores os quatro fatores de ordem superior (abertura à mudança, conservação, autotranscendência e autopromoção) foi utilizada para verificar o valor preditivo dos valores sobre o comportamento de usar preservativo no relacionamento sexual. Antes de proceder ao cálculo da regressão múltipla foi verificado se os dados atendiam os diversos critérios apresentados por Tabachnick e Fidell (1989) para análises multivariadas. Em conseqüência desta análise, foram eliminados os outliers bem como os casos nos quais algum item tinha ficado sem resposta. Desta forma, a amostra para a regressão múltipla foi de 244. Obviamente foi também verificado se a amostra assim reduzida atendia adequadamente as exigências para garantir a consistência dos resultados obtidos. Segundo Tabachnick e Fidell (1989), quando se utiliza regressão múltipla stepwise a relação entre os sujeitos e os preditores deve ser de, no mínimo, 40 sujeitos para cada VI. Na presente pesquisa esta relação foi de 60 para 1.

\section{Resultados}

O uso de preservativo relacionou-se negativamente com as crenças "os preservativos diminuem a sensação sexual" $(-0,22 ; p<0,01)$ e propor ao parceiro(a) o uso de camisinha "demostra falta de confiança nele(a)" (-0,20; $p<0,01)$ e positivamente com a opinião que quem estima a sua saúde deve usar camisinha regularmente $(0,15 ; p<$ $0,05)$. As correlações bivariadas entre cada um dos tipos motivacionais de valores e a freqüência de uso de preservativo encontram-se na Tabela 2. Hedonismo, estimulação, autodeterminação e realização correlacionaram-se positivamente com o uso de preservativo e

Tabela 2. Correlações Bivariadas entre os TMV e o Uso de Preservativo

\begin{tabular}{lc}
\hline Tipos Motivacionais de Valores & Correlação \\
\hline Segurança & $-0,01$ \\
Conformidade & 0,02 \\
Tradição & $-0,17^{*}$ \\
Benevolência & 0,03 \\
Universalismo & 0,02 \\
Autodeterminação & $0,15^{*}$ \\
Estimulação & $0,20^{* *}$ \\
Hedonismo & $0,23^{* * *}$ \\
Realização & $0,17^{*}$ \\
Poder & 0,06 \\
\hline
\end{tabular}

$* p<0,05 \quad * * p<0,01 \quad * * * p<0,001$ 
tradição negativamente. Os outros TMV apresentaram correlações nulas ou muito baixas. A relação dos TMV com o uso de preservativo apresentou uma curva sinusóide, mas não simétrica. Os resultados da análise de regressão múltipla mostraram que somente a abertura à mudança entrou no modelo como regressor, os outros três fatores foram excluídos. $R^{2}$ foi igual a 0,04 e $\beta$ igual a 0,20 (Tabela 3). excluídos. Apesar da força preditiva da abertura ser fraca $\left(R=0,20, R^{2}=0,04\right)$, este resultado é importante porque revela uma relação positiva entre a prioridade dada aos valores de abertura à mudança e o uso de preservativo $(b=0,20)$. O uso de preservativo, certamente, é um comportamento complexo, determinado por múltiplos fatores psicossociais, cada um deles explicando somente pequenas porcentagens do mesmo. A introdução de

Tabela 3. Resultados da Análise de Regressão Múltipla Stepwise para o Uso de Preservativo

\begin{tabular}{|c|c|c|c|c|c|c|c|}
\hline \multicolumn{2}{|c|}{ Regressão múltipla ${ }^{\mathrm{b}}$} & \multicolumn{5}{|c|}{ Análise da variancia } & \multirow[b]{2}{*}{$p$} \\
\hline$R$ & 0,199 & & $S Q$ & $d f$ & $M Q$ & $F$ & \\
\hline$R^{2}$ & 0,040 & Regressão & 16,916 & 1 & 16,916 & 9,987 & $0,002^{a}$ \\
\hline$R^{2}$ ajustado & 0,036 & Resíduo & 409,916 & 242 & 1,694 & & \\
\hline Erro padrão & 1,300 & Total & 426,832 & 243 & & & \\
\hline
\end{tabular}

a. Preditor: Abertura à mudança b. Variável dependente: Uso de preservativo

\section{Discussão}

Como explicar os resultados observados? Convém destacar que os TMV com correlações positivas e negativas em relação ao uso de preservativo situam-se na dimensão abertura à mudança versus conservação. O TMV conformidade, segurança e tradição constituem o pólo da conservação. Os valores que formam o TMV tradição têm como meta a aceitação e o comprometimento com os costumes e as idéias da cultura tradicional e da religião. Como foi discutido na introdução, o uso de preservativo não é um comportamento que faz parte das tradições culturais do país. Pelo contrário, ele é novidade e oposto aos costumes e formas de pensar tradicionais. Além disso, o uso de preservativo é objetivo de interdição da religião católica que é dominante no país. A correlação negativa do TMV tradição com o uso de preservativo pode-se explicar pelo fato deste comportamento ser alheio às tradições culturais do país.

Os tipos motivacionais hedonismo, autodeterminação e estimulação constituem os componentes do pólo de abertura à mudança que tem como meta a procura de caminhos novos, mesmo se incertos, opostos ou diferentes das formas tradicionais de agir e de pensar. $\mathrm{O}$ uso de preservativo constitui um comportamento novo, uma mudança importante nas formas tradicionais do relacionamento sexual. Isto explica porque a freqüência do uso de preservativo aumenta quando a prioridade aos valores de hedonismo, autodeterminação e estimulação aumenta. A abertura à mudança parece constituir um elemento importante no uso de preservativo pelos jovens universitários.

Somente a abertura à mudança entrou no modelo como regressor, os outros três fatores tendo sido preservativo no encontro sexual é um comportamento inesperado, não prescrito pelas normas tradicionais que regem este tipo de atividade e que exige, em conseqüência, uma motivação ou interesse da parte da pessoa para quebrar o status quo e orientar o seu comportamento por um caminho novo que, por ser novo, pode ser incerto. É, precisamente, o sentido da motivação axiológica representada pela abertura à mudança.

Numa cultura de tipo tradicionalista e bastante influenciada pelas normas da religião católica, como é a brasileira (Ubillos, Paez \& González, 2000), os resultados desta pesquisa podem ser importantes para melhor compreender e explicar o insucesso relativo de campanhas para o uso preventivo de preservativo nas relações sexuais bem como para, na elaboração de futuros programas preventivos, tentar analisar e levar em consideração um maior número de determinantes do uso de preservativo.

Uma limitação desta pesquisa é relacionada com a generalização dos resultados. A amostra foi composta por estudantes universitários de uma universidade pública. Apesar de considerar que os resultados podem se generalizar para estudantes universitários com características demográficas semelhantes, não é evidente se eles podem ser generalizados para adolescentes, adultos e indivíduos de nível sócio-econômico inferior.

Concluindo, as prioridades axiológicas da pessoa constituem um fator psicossocial preditivo do uso de preservativo, que se manifesta, positiva e principalmente, pela importância dada aos TMV hedonismo, estimulação e autodeterminação e, negativamente, pela ênfase dada ao TMV tradição. Como foi salientado na introdução, diversos antecedentes do uso de preservativo já têm sido identificados pelos pesquisadores e, certamente, outros 
preditores ainda poderão ser identificados. A contribuição das prioridades axiológicas para a compreensão deste comportamento é modesta, mas ela pode ser valiosa na elaboração de programas preventivos.

\section{Referências}

Arruda, J. M., Morris, L. \& Rutenberg, N. (1987). Pesquisa nacional sobre saúde materno infantil e planejamento familiar. BemFam, Agosto, 107109.

Bayés, R. (1992). Aportaciones del análisis funcional de la conducta al problema del SIDA. Revista Latinoamericana de Psicologia, 24(1-2), 35-56.

Biernat, M., Theno, S. A. \& Crandall, C. S. (1996). Values and prejudice: Toward understanding the impact of american values on outgroup attitudes. Em C. Seligman, J. M. Olson \& M. P. Zanna (Orgs.), The Psychology of values: The Ontario Psymposium (Vol. 8, pp. 153-189). Mahwah, N. J.: Laurence Erlbaum Associates.

Bryan, A. D., Aiken, L. S. \& West, S. G. (1996). Increasing condom use: Evaluation of a theory-based intervention to prevent sexually transmitted diseases in young women. Health Psychology, 15, 371-382.

Bryan, A. D., Aiken, L. S. \& West, S. G. (1999). The impact of males proposing condom use on perceptions of an initial sexual encounter. Personality and Social Psychology Bulletin, 25(3), 275-286.

Campbell, S. M., Peplau, L. A. \& DeBro, S. C. (1992). Women, men and condoms: Attitudes and experiences of heterosexual college students. Psychology of Women Quarterly, 16, 273-288.

De Dreu, C. K. W. \& Van Lange, P. A. M. (1995). The impact of social value orientations on negotiator cognition and behavior. Personality and Social Psychology Bulletin, 21, 1178-1188.

Emmons, C. A., Joseph, J. G., Kressler, R. C., Montgomery, S. B. \& Ostrow, D. G. (1986). Psychological predictors of reported behavior change in homosexual men at risk for AIDS. Health Education Quarterly, 13(4), 331-345.

Feather, N. T. (1970). Educational choice and students attitudes in relation to terminal and instrumental values. Australian Journal of Psychology, 22, 177-144.

Feather, N. T. (1995). Values, valences, and choice: The influences of values on the perceived attractiveness and choice of alternatives. Journal of Personality and Social Psychology, 68(6), 1135-1151.

Ferraz, E. A., Ferreira, I. Q., Rutenberg, N. \& Soares, M. P. (1992). Pesquisa sobre saúde familiar no Nordeste do Brasil - 1991. BemFam, Março, 7678.

Fisher, J. D. \& Misovich, S. J. (1990). Evolution of college students' AIDSrelated behavioral responses, attitudes, knowledge and fear. AIDS education and prevention, 2(4), 322-337.

Gimenes, M. d. G. G., Pedrazani, E., Basso, A. F. T., Pontes, A. C., Marque, C. d. R., De Souza, D. M. X., Maldonado, D. P. A., Bertuso, E. C., Da Silva, E. L., Consoni, E. B., Romão, D. d. C. \& Banhos, E. (1996). A prevenção da AIDS entre estudantes universitários: A resposta da UFSCAR. Temas em Psicologia, (1), 59-69.

Greenstein, T. N. (1976). Behavior change through value self-confrontation: A field experiment. Journal of Personality and Social Psychology, 34, 254262.

Helweg-Larsen, M. \& Collins, B. E. (1994). The UCLA multidimensional condom attitudes scale: Documenting the complex determinants of condom use in college students. Health Psychology, 13, 224-237.

Homant, R. \& Rokeach, M. (1970). Values for honesty and cheating behavior. Personality, 1, 153-162.

Hynie, M. \& Lydon, J. E. (1995). Women's perceptions of female contraceptive behavior: Experimental evidence of the sexual double standard. Psychology of Women Quarterly, 19, 563-581.

Kahle, L. R. (1996). Social values and consumer behavior: Research from the List of Values. Em C. Seligman, J. M. Olson, \& M. P. Zanna (Orgs.), The Psychology of values: The Ontario Symposium (Vol. 8, pp. 135-151). Mahwah, N. J: Laurence Erlbaum Associates.
Kelly, J. A., Lawrence, J. S. \& Brasfield, T. L. (1991). Predictors of vulnerability to AIDS risk behavior relapse. Journal of Consulting and Clinical Psychology, 59(1), 163-166.

Rokeach, M. (1969). Value systems in religion. Review of Religious Research, 11, 3-23.

Rokeach, M. (1973). The nature of human values. New York: Free Press.

Roscoe, B. \& Kruger, T. L. (1990). AIDS: Late adolescent's knowledge and its influence on sexual behavior. Adolescence, 25, 39-48.

Sagiv, L. \& Schwartz, S. H. (1995). Value priorities and readiness for outgroup social contact. Journal of Personality and Social Psychology, 69(3), 437-448.

Schwartz, S. H. (1992). Universals in the content and structure of values: Theoretical advances and empirical tests in 20 countries. Em M. Zanna (Org.), Advances in Experimental Social Psychology (Vol. 25, pp. 165). Orlando: Academic.

Schwartz, S. H. (1994). Are there universal aspects in the structure and contents of human values? Journal of Social Issues, 50(4), 19-45.

Schwartz, S. H. (1996). Value priorities and behavior: Applying a theory of integrated value systems. Em C. Selligman, J. M. Olson \& M. P. Zanna (Org.), The Psychology of Values:The Ontario Symposium (Vol. 8, pp. 1-24). Ontario: Mahwah.

Schwartz, S. H. \& Bilsky, W. (1987). Toward a universal psychological structure of human values. Journal of Personality and Social Psychology, 53(3), 550-562.

Schwartz, S. H. \& Bilsky, W. (1990). Toward a theory of the universal content and structure of values: Extensions and cross-cultural replications. Journal of Personality and Social Psychology, 58(5), 878-891.

Schwartz, S. H. \& Huismans, S. (1995). Value priorities and religiosity in four Western religions. Social Psychology Quarterly, 58(2), 88-107.

Shayer, B. P. M. (1994). Fatores psicossociais preditivos de freqüência de uso de preservativo em resposta à epidemia da AIDS. Dissertação de Mestrado nãopublicada, Curso de Pós-Graduação em Psicologia, Universidade de Brasília, Brasília, DF.

Shotland, R. L. \& Berger, W. G. (1970). Behavioral validation of several values from the Rokeach value scale as an index of honesty. Journal of Applied Psychology, 54, 433-435.

Tabachnick, B. G. \& Fidell, L. S. (1989). Using multivariate statistics. New York: HarperCollins.

Tamayo, A. (1994). Hierarquia de valores transculturais e brasileiros. Psicologia: Teoria e Pesquisa, 10(2), 269-285.

Tamayo, A. \& Schswartz, S. H. (1993). Estrutura motivacional dos valores humanos. Psicologia: Teoria e Pesquisa, 9(2), 329-348.

Thurman, Q. C. \& Franklin, K. M. (1990). AIDS and college health: Knowledge, treat and prevention at a Northeastern University. Journal of American College Health, 38, 179-184.

Ubillos, S, Páez, D. \& González, J. L. (2000). Culture and sexual behavior. Psychothema, 12, 70-82.

Valdisserri, R. O., Lyter, D., Leviton, L. C., Callahan, C. M., Kingsley, L. A. \& Rinaldo, C. R. (1988). Variables influencing condom use in a cohort of gay and bisexual men. American Journal of Public Health, 78(7), 801805.

Walter, H., Vaughn, R. D., Ragin, D. F., Cohall, A. T. \& Kasen, S. (1994). Prevalence and correlates of AIDS-related intentions among urban minority high school students. AIDS Education and Prevention, 6, 339350.

Wilson, D., Manual, A. \& Lavelle, S. (1991). Psychological predictors of condom use to prevent HIV transmission among Zimbabwean. International Journal of Psychology, 26(6), 705-721.

Wulfert, E. \& Wan, C. K. (1993). Condom use: A self-efficacy model. Health Psychology, 12, 346-353.

Recebido em 29/05/2000

Revisado em 13/09/2000

Aceito em13/09/2000 
Sobre os autores:

Alvaro Tamayo é professor titular do Departamento de Psicologia Social e do Trabalho da Universidade de Brasília. Foi professor da Université de Moncton (Canadá). Doutor em Psicologia Social pela Université de Louvain (Bélgica).

Adilce Lima é assistente social formada pela Universidade de Brasília.

Julina Marques é assistente social formada pela Universidade de Brasília.

Larissa Martins é assistente social formada pela Universidade de Brasília. 